\title{
Dynamics of Neutrophils-to-Lymphocyte Ratio Predict Outcomes of PD-1/PD-L1 Blockade
}

\author{
Michele Moschetta, ${ }^{1}$ Mario Uccello, ${ }^{1}$ Benjamin Kasenda, ${ }^{2}$ \\ Gabriel Mak, ${ }^{1}$ Anissa McClelland, ${ }^{1,3}$ Stergios Boussios, ${ }^{4}$ \\ Martin Forster, ${ }^{3}$ and Hendrik-Tobias Arkenau ${ }^{1,3}$ \\ ${ }^{1}$ Drug Development Unit, Sarah Cannon Research Institute, London, UK \\ ${ }^{2}$ University Hospital of Basel, Basel, Switzerland \\ ${ }^{3}$ The University College London Cancer Institute, London, UK \\ ${ }^{4}$ Department of Medical Oncology, University of Ioannina, Ioannina, Greece \\ Correspondence should be addressed to Michele Moschetta; michelemoschettal@gmail.com
}

Received 20 August 2017; Accepted 5 November 2017; Published 28 November 2017

Academic Editor: Ilaria G. Zizzari

Copyright ( 2017 Michele Moschetta et al. This is an open access article distributed under the Creative Commons Attribution License, which permits unrestricted use, distribution, and reproduction in any medium, provided the original work is properly cited.

\begin{abstract}
Introduction. Baseline neutrophil-to-lymphocyte ratio (NLR) has been repeatedly reported as a significant prognostic factor in advanced cancer patients. We explored whether changes in NLR may predict outcome of advanced cancer patients enrolled into phase 1 trials and treated with PD-1/PD-L1 inhibitors. Patients and Methods. Advanced cancer patients enrolled into phase 1 trials between September 2013 and May 2016 and treated with anti-PD-1/PD-L1 agents were included in this retrospective study. NLR was calculated at baseline and after 2 cycles of treatment. Royal Marsden Hospital (RMH) prognostic score and Eastern Cooperative Group (ECOG) performance status (PS) were determined at baseline. Kaplan-Meier estimation and Cox regression analyses were used to assess the impact of NLR dynamics on PFS. Results. Among the 55 patients eligible, 26 (47\%) were treated with anti-PDL1 monotherapy, 22 (40\%) received single agent anti-PD-1, and 7 (13\%) were given a tyrosine kinase inhibitor (TKI) plus a PD-1 inhibitor. Neither ECOG PS nor RMH prognostic score was significantly associated with PFS in our cohort, whereas changes in NLR significantly impacted on PFS. Conclusion. Changes in the NLR may be a useful predicting factor in advanced cancer patients treated with anti-PD-1/PD-L1 agents. Further prospective trials are needed to verify these findings.
\end{abstract}

\section{Introduction}

Immune checkpoint inhibitors have emerged as potent and effective treatments for various types of haematological and solid malignancies [1]. In particular, blockade of the PD1/PD-L1 axis can result in dramatic and sustained tumour regression in multiple cancer types $[2,3]$. Under normal circumstances, this pathway is necessary to maintain immune homeostasis [4]. When PD-L1 binds to PD-1, an inhibitory signal is transmitted into the T-cell, protecting normal cells from collateral damage. Nevertheless, upregulation of PDL1 may allow cancer cells to evade immune surveillance [3]. Considering the costs and potential side effects of novel anti-PD-1/PD-L1 agents, it is of vital importance to identify reliable biomarkers to select the most suitable patients for these drugs while sparing nonresponders from toxicity.

$\mathrm{PD}-\mathrm{L} 1$ expression as determined by immunohistochemistry is considered the most useful biomarker in predicting outcomes of PD-1/PD-L1 blockade [4]. Several studies have investigated the role of $\mathrm{PD}-\mathrm{L} 1$ expression in tumour and stromal cells as a potential biomarker of response, but the results were somewhat contradictory $[4,5]$. Indeed, several factors can limit the reliability of this biomarker, including the use of different monoclonal antibodies for detection of PD-L1, variable procedures for biopsy collection and storage, lack of defined thresholds to describe PD-L1 expression in samples, and intratumour heterogeneity in PD-L1 expression [5] The presence of microsatellite instability, tumour 
mutational load, tumour-infiltrating lymphocytes (TILs), myeloid-derived suppressor cells (MDSCs), indoleamine 2,3dioxygenase, regulatory $\mathrm{T}$ cells, and immune specific signatures have been also investigated with promising results [68]. Despite the aforementioned methods, there is still a lack of a simple, effective, and definitive biomarker of response to immune checkpoint inhibitors.

Increased neutrophil-to-lymphocyte ratio (NLR) has been reported as an independent poor prognostic indicator in several malignancies and its normalisation following treatment has been found to predict survival in cancer patients considered for early phase clinical trials [9]. Here, we investigated the usefulness of NRL changes in predicting progression-free survival (PFS) in patients undergoing treatment with $\mathrm{PD}-1 / \mathrm{PD}-\mathrm{L} 1$ inhibitors within phase 1 clinical trials.

\section{Patients and Methods}

Data of metastatic cancer patients enrolled in phase 1 trials between September 2013 and May 2016 in our institution were retrospectively reviewed. Patients treated with PD-1/PD-L1 checkpoint-directed therapy were eligible. All the subjects had a histologically confirmed diagnosis of metastatic solid cancer and were intended to receive treatment with an antiPD-1/PD-L1 agent given as monotherapy or in combination with a tyrosine kinase inhibitor (TKI). Baseline parameters, tumour characteristics, and treatment data were all reviewed and anonymously collected for this study. All the subjects met the standard inclusion criteria for phase 1 trials: Eastern Cooperative Group (ECOG) performance status (PS) 0 or 1; measurable disease based on Response Evaluation Criteria in Solid Tumour (RECIST); adequate bone marrow, liver, and kidney function; life expectancy of at least 3 months. Baseline characteristics recorded in the eligible population included demographic variables, tumour type, anticancer treatment (anti-PD-1 versus anti-PD-L1 versus anti-PD-L1 plus TKI), number of previous lines for metastatic disease, Royal Marsden Hospital (RMH) prognostic score [10], white blood cell (WBC) level, absolute neutrophil count (ANC), absolute lymphocyte count (ALC), and neutrophil-to-lymphocyte ratio (NLR). The RMH prognostic score (range $0-3$ ) was calculated at baseline, taking into account albumin level, lactate dehydrogenase (LDH) level, and number of metastatic sites [10]. The NLR was calculated using the standard formula: NLR $=$ ANC/ALC. NLR was calculated at baseline (cycle 1 day 1), and after 6 weeks (2 cycles) of treatment. Patients were treated until disease progression, death, or unacceptable toxicity. We considered PFS as our main outcome, which was defined as the time from treatment start until progression or death, whichever occurred first.

To investigate the dynamics in NLR between baseline and after 2 cycles of anti-PD-1/PD-L1 therapy, we used a landmark approach by excluding patients who were not able to receive at least 2 cycles of treatment to avoid guarantee time bias. We used multivariate Cox regression analyses with the relative NLR difference as independent and PFS as the dependent variable. To adjust for possible confounding, we
TABLE 1: Patients' characteristics at baseline. NCSLC = non-small cell lung cancer; ECOG PS = Eastern Cooperative Oncology Group performance status; GI = gastrointestinal; TKI = tyrosine kinase inhibitor; RMH = Royal Marsden Hospital.

\begin{tabular}{lc}
\hline Characteristic & $n(\%)$ \\
\hline Sex & \\
Male & $19(35)$ \\
Female & $36(65)$ \\
ECOG PS & \\
0 & $36(65)$ \\
1 & $19(35)$ \\
$>1$ & - \\
Tumour type & \\
NSCLC & $18(33)$ \\
Upper GI cancer & $11(20)$ \\
Bladder cancer & $8(15)$ \\
Renal cell carcinoma & $8(15)$ \\
Breast cancer & $7(13)$ \\
Colorectal cancer & $2(4)$ \\
Ovarian cancer & $1(2)$ \\
Therapy & \\
Anti-PD-1 & $22(40)$ \\
Anti-PD-L1 & $26(47)$ \\
Anti-PD-L1 plus TKI & $7(13)$ \\
RMH prognostic score & \\
0 & $31(56)$ \\
1 & $19(35)$ \\
2 & $3(5)$ \\
3 & $2(4)$ \\
Age & $2(1-4)$ \\
Number of metastatic sites & $1(1-6)$ \\
Number of previous treatment lines & \\
\hline & \\
&
\end{tabular}

introduced the RMH score into the model and additionally added a random effect for tumour entity, in order to account for possible heterogeneity between tumour types. We calculated univariate and multivariate hazard ratios (HR) with accompanied 95\% confidence intervals (CI); however, the multivariable analysis is considered as main analysis. To visualize the prognostic effect of the NLR difference, we created Kaplan-Meier plots. All $p$ values are exploratory in nature with a conventional level of significance at 0.05 . All analyses were done using the statistical software $\mathrm{R}$ (https://www.r-project.org/) and STATA (version 14).

\section{Results}

A total of 67 potentially eligible patients were identified. Of those, 12 subjects received less than 2 cycles and were therefore excluded from the analysis. The characteristics of the included 55 patients are summarised in Table 1. Median age of patients included was 61 years (40 to 80 years). The most represented tumour type was non-small cell lung cancer (NSCLC) with 18 (33\%) subjects, followed by upper 
TABLE 2: Distribution of patient population in two groups. Group A: neutrophil-to-lymphocyte ratio (NLR) after 2 cycles $\leq$ median baseline NLR. Group B: NLR after 2 cycles $>$ median baseline NLR. ECOG PS = Eastern Cooperative Oncology Group performance status; $\mathrm{RMH}=$ Royal Marsden Hospital; $\mathrm{ANC}=$ absolute neutrophil count; ALC = absolute lymphocyte count; $\mathrm{SD}=$ standard deviation; $\mathrm{IQR}$ = interquartile range; NLR = neutrophil-to-lymphocyte ratio.

\begin{tabular}{|c|c|c|}
\hline Characteristic & $\begin{array}{l}\text { Group A } \\
(n=28)\end{array}$ & $\begin{array}{l}\text { Group B } \\
(n=27)\end{array}$ \\
\hline \multicolumn{3}{|l|}{$\operatorname{Sex} n(\%)$} \\
\hline Female & $11(39)$ & $8(30)$ \\
\hline Male & $17(61)$ & $19(70)$ \\
\hline \multicolumn{3}{|l|}{ ECOG PS $n(\%)$} \\
\hline 0 & $16(43)$ & $20(74)$ \\
\hline 1 & $12(57)$ & $7(26)$ \\
\hline \multicolumn{3}{|c|}{ RMH prognostic score $n(\%)$} \\
\hline $0-1$ & $25(89)$ & $25(93)$ \\
\hline $2-3$ & $3(11)$ & $2(7)$ \\
\hline \multicolumn{3}{|l|}{ Intervention $n(\%)$} \\
\hline Anti-PD-1 & $14(50)$ & $8(30)$ \\
\hline Anti-PD-L1 & $9(32)$ & $17(63)$ \\
\hline Anti-PD-1 plus TKI & $5(18)$ & $2(7)$ \\
\hline \multicolumn{3}{|l|}{ Baseline ANC } \\
\hline Mean (SD) & $5.1(1.8)$ & $4.1(1.4)$ \\
\hline \multicolumn{3}{|l|}{ Baseline ALC } \\
\hline Mean (SD) & $1.3(0.6)$ & $1.3(0.5)$ \\
\hline \multicolumn{3}{|l|}{ Baseline NLR } \\
\hline Median (IQR) & $3.9(2.7-5.6)$ & $3.0(2.5-4.8)$ \\
\hline \multicolumn{3}{|l|}{ ANC after 2 cycles } \\
\hline Median (IQR) & $3.7(2.8-5.1)$ & $4.5(3.5-5.7)$ \\
\hline \multicolumn{3}{|l|}{ ALC after 2 cycles } \\
\hline Mean (SD) & $1.4(0.5)$ & $1.2(0.5)$ \\
\hline \multicolumn{3}{|l|}{ NLR after 2 cycles } \\
\hline Median (IQR) & $2.9(2.2-3.7)$ & $3.9(2.8-7)$ \\
\hline
\end{tabular}

gastrointestinal ( $n=10 ; 18 \%)$, bladder $(n=8 ; 15 \%)$, and breast $(n=7 ; 13 \%)$ carcinomas. Median number of previous lines of treatment for metastatic disease was 1 (range 1-6) while baseline median number of metastatic sites of disease was 2 (range 1-4). RMH prognostic score at baseline was 0 in $31(56 \%)$ subjects and 1 or higher in $24(64 \%)$ subjects. Proportion of patients with ECOG PS 0 or 1 was $36(65 \%)$ and 19 (35\%), respectively. In total, 26 (47\%) of the patients were treated with anti-PD-L1 monotherapy, 22 (40\%) received single agent anti-PD-1, and $7(13 \%)$ were given a TKI in combination with a PD-1 inhibitor.

On univariate analysis, baseline NLR, treatment modality, RMH score, ECOG PS, and number of metastatic sites did not have significant impact on PFS. Baseline median NLR was 3.4 in the overall population. Patient population was divided into 2 distinct groups based on decrease (Group A) or increase (Group B) of NLR in comparison with median NLR after treatment with a PD-1/PD-L1 inhibitor. No substantial differences in distribution were observed between these 2 groups in terms of age, sex, type of treatment, ECOG PS, and $\mathrm{RMH}$ prognostic score (Table 2). Increased NLR after 2 cycles of anti-PD-1/PD-L1 therapy had a negative effect on PFS (HR 1.14, 95\% CI 1.06-1.23, $p=0.004$ ), (Figure 1). This effect was also observed in our multivariate analysis,

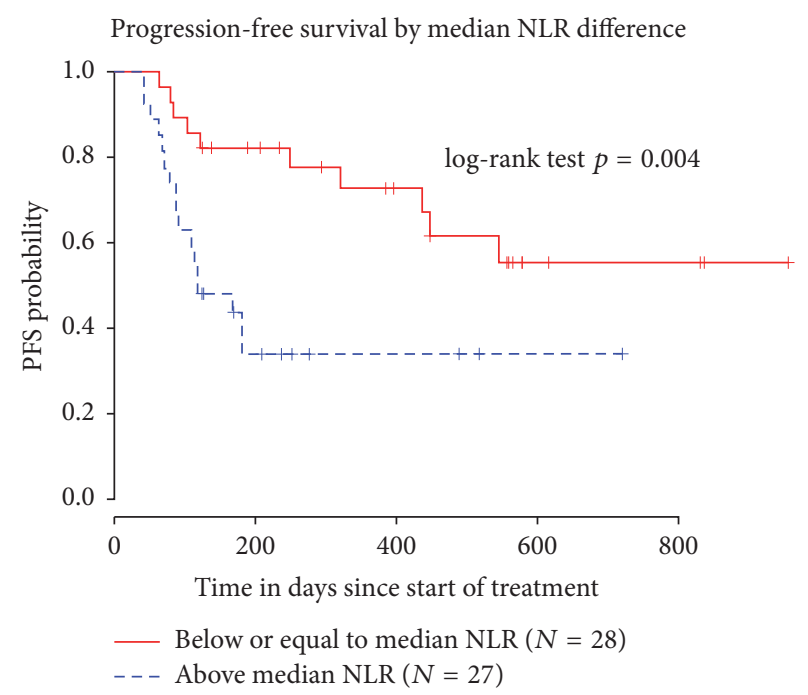

FIgURe 1: Progression-free survival (PFS) stratified by median differences in neutrophil-to-lymphocyte ratio (NLR) between baseline and after 2 doses of treatment with an anti-PD-1/PD-L1 inhibitor, showing longer PFS in patients with a reduction of NLR compared to the median baseline NLR.

where increased NLR was associated with decreased PFS after adjusting for RMH prognostic score (HR 1.03, 95\% CI 1.01-1.04, $p<0.001)$. Baseline median ANC level was significantly higher in Group A than in Group B $(p=0.029)$. In Group A, a reduction in median ANC was shown after 2 cycles of treatment when compared with baseline ANC level, whereas this was not observed in Group B. After 2 cycles of treatment with anti-PD-1/PD-L1 agent, median ANC was significantly higher in Group B when compared to Group A $(p=0.014)$. Median ALC did not change significantly after treatment $(p=0.222)$ and no significant differences were shown between baseline and posttreatment values in both Group A and Group B ( $p=0.24)$ (Table 2).

\section{Discussion}

Molecular selection in patients undergoing treatment with immune checkpoint inhibitors is an urgent unmet medical need. Ongoing approval of several anti-PD-1/PD-L1 agents and the emergence of safety concerns from immune-related adverse events also highlight the need for biomolecular stratification. Several biomarkers have been investigated, some of which have shown potential usefulness in predicting the activity of these agents. So far, PD-L1 expression in tumour cells remains the most reliable but many technical limitations have been associated with this biomarker $[4,5]$. Furthermore, individuals with negative PD-L1 expression can still respond to PD-1/PD-L1 blockade, further questioning the value of PD-L1 expression as a universal biomarker [11]. Therefore, alternative markers of response need to be identified.

Clinicopathologic factors have been extensively investigated in several tumour types and anticancer therapies. Among them, ECOG PS has been repeatedly reported as a strong predictor of survival in multiple settings. The RMH 
prognostic score was electively implemented in advanced cancer patients enrolled in phase 1 studies [10]. Unexpectedly, neither ECOG PS nor RMH prognostic score were significantly associated with PFS in our cohort. Our results are not consistent with previous studies showing significant prognostic significance of RMH score and ECOG PS in phase 1 trial patients $[10,12,13]$.

Baseline NLR has been reported to predict overall survival in cancer patients undergoing both conventional chemotherapy and targeted treatments, including immune checkpoint inhibitors. In previous reports, a correlation between baseline NLR and survival was shown in kidney cancer and NSCLC treated with IL-2 and nivolumab, respectively $[14,15]$. Conversely, in our group of patients, baseline NLR was not found to correlate with PFS, although this result may have been determined by the limited sample size. In our study, we retrospectively analysed phase 1 trial cancer patients with advanced disease who had received at least 2 cycles of treatment with anti-PD-1/PD-L1 agent, to assess the significance of NLR as an independent biomarker in predicting clinical benefit in terms of PFS. Interestingly, a decrease in NLR after 2 cycles of treatment with PD-1/PD-L1 blockade was associated with longer PFS in our cohort. Accordingly, changes in NLR had shown to predict better outcomes in cancer patients undergoing conventional chemotherapy or targeted treatment but had been never investigated in subjects receiving immune checkpoint inhibitors [16-18].

Another important finding in this study was the observation that negative or positive changes in NLR were driven by a decrease in ANC and not by changes in ALC as one would have expected. Though retrospective in nature, our findings on ANC may be interpreted as hypothesisgenerating. Despite the fact the $\mathrm{T}$ lymphocyte activity is the main target of PD-1/PD-L1 blockade, our results may suggest an important interaction between the neutrophils and tumour microenvironment. We may also speculate that the systemic effect of anti-PD-1/PD-L1 agents involves a crucial effect on circulating myeloid populations included in the ANC as measured by automated full blood cell count analyser. Preclinical evidence shows that MDSCs can impair the efficacy of immunotherapy [19]. Under physiological conditions, there is a low level of MDSCs in the bloodstream while these populations rapidly expand during immunological responses to infections, inflammation, and cancer [20]. MDSCs can adopt multiple mechanisms to induce immunosuppression, including production of arginase 1 and inducible nitric oxide synthase, leading to T-cell inhibition $[19,20]$. MDSCs are also known to enhance cancer cell proliferation, confer resistance to anticancer therapies, and promote angiogenesis and metastasis [20]. Concomitant targeting of MDSCs may therefore increase the antitumour activity of PD-1/PD-L1 inhibitors in nonresponders. Moreover, a decreased mobilisation of MDSCs from the bone marrow may represent a systemic effect of anti-PD-1/PD-L1 treatment that needs to be better investigated in preclinical studies. A subsequent decrease of tumour-infiltrating MDSCs may then unleash antitumour activity of TILs and ultimately contribute to the therapeutic effect of anti-PD-1/PD-L1 agents.
Our study has several biases, including its retrospective nature, limited sample size, heterogeneous tumour types, and the choice of PFS as endpoint instead of overall survival. Nevertheless, we showed that NLR, a simple haematological parameter easily obtainable in daily clinical practice, may be used to predict clinical benefit from PD-1/PD-L1 inhibitors. These results are in line with common clinical experience with these agents, where a rapid clinical benefit can be observed despite unusual initial patterns of imaging response. Further studies conducted in larger prospective cohorts of patients undergoing treatment with immune checkpoint inhibitors are needed to confirm the predictive role of NLR in this setting.

\section{Conflicts of Interest}

The authors declare no conflicts of interest.

\section{Authors' Contributions}

Michele Moschetta, Mario Uccello, and Benjamin Kasenda equally contributed to the article.

\section{Acknowledgments}

The authors are grateful to the patients and their families for participating in these clinical studies.

\section{References}

[1] A. Goodman, S. P. Patel, and R. Kurzrock, "PD-1-PD-L1 immune-checkpoint blockade in B-cell lymphomas," Nature Reviews Clinical Oncology, 2016.

[2] J. Hamanishi, M. Mandai, N. Matsumura, K. Abiko, T. Baba, and I. Konishi, "PD-1/PD-L1 blockade in cancer treatment: perspectives and issues," International Journal of Clinical Oncology, vol. 21, no. 3, pp. 462-473, 2016.

[3] D. M. Pardoll, "The blockade of immune checkpoints in cancer immunotherapy," Nature Reviews Cancer, vol. 12, no. 4, pp. 252 264, 2012.

[4] V. A. Boussiotis, "Molecular and biochemical aspects of the PD1 checkpoint pathway," The New England Journal of Medicine, vol. 375, no. 18, pp. 1767-1778, 2016.

[5] S. L. Topalian, J. M. Taube, R. A. Anders, and D. M. Pardoll, "Mechanism-driven biomarkers to guide immune checkpoint blockade in cancer therapy," Nature Reviews Cancer, vol. 16, no. 5, pp. 275-287, 2016.

[6] J. Galon, A. Costes, F. Sanchez-Cabo et al., “Type, density, and location of immune cells within human colorectal tumors predict clinical outcome," Science, vol. 313, no. 5795, pp. 19601964, 2006.

[7] R. B. Holmgaard, D. Zamarin, A. Lesokhin, T. Merghoub, and J. D. Wolchok, "Targeting myeloid-derived suppressor cells with colony stimulating factor- 1 receptor blockade can reverse immune resistance to immunotherapy in indoleamine 2,3dioxygenase-expressing tumors," EBioMedicine, vol. 6, pp. 50$58,2016$.

[8] W. Joost Lesterhuis, A. Bosco, M. J. Millward, M. Small, A. K. Nowak, and R. A. Lake, "Dynamic versus static biomarkers in 
cancer immune checkpoint blockade: Unravelling complexity," Nature Reviews Drug Discovery, vol. 16, no. 4, pp. 264-272, 2017.

[9] D. J. Pinato, C. Stavraka, M. J. Flynn et al., "An inflammation based score can optimize the selection of patients with advanced cancer considered for early phase clinical trials," PLoS ONE, vol. 9, article e83279, no. 1, 2014.

[10] H.-T. Arkenau, J. Barriuso, D. Olmos et al., "Prospective validation of a prognostic score to improve patient selection for oncology phase I trials," Journal of Clinical Oncology, vol. 27, no. 16, pp. 2692-2696, 2009.

[11] C. Grigg and N. A. Rizvi, "PD-L1 biomarker testing for nonsmall cell lung cancer: Truth or fiction?” Journal for ImmunoTherapy of Cancer, vol. 4, no. 1, article 48, 2016.

[12] I. Garrido-Laguna, F. Janku, C. Vaklavas et al., "Validation of the Royal Marsden Hospital prognostic score in patients treated in the phase i clinical trials program at the MD Anderson Cancer Center," Cancer, vol. 118, no. 5, pp. 1422-1428, 2012.

[13] T. Kawaguchi, M. Takada, A. Kubo et al., "Performance status and smoking status are independent favorable prognostic factors for survival in non-small cell lung cancer: A comprehensive analysis of 26,957 patients with NSCLC," Journal of Thoracic Oncology, vol. 5, no. 5, pp. 620-630, 2010.

[14] J. A. Kuzman, D. D. Stenehjem, J. Merriman et al., "Neutrophillymphocyte ratio as a predictive biomarker for response to high dose interleukin-2 in patients with renal cell carcinoma," $B M C$ Urology, vol. 17, article 1, no. 1, 2017.

[15] S. J. Bagley, S. Kothari, C. Aggarwal et al., "Pretreatment neutrophil-to-lymphocyte ratio as a marker of outcomes in nivolumab-treated patients with advanced non-small-cell lung cancer," Lung Cancer, vol. 106, pp. 1-7, 2017.

[16] W. Chua, K. A. Charles, V. E. Baracos, and S. J. Clarke, "Neutrophil/lymphocyte ratio predicts chemotherapy outcomes in patients with advanced colorectal cancer," British Journal of Cancer, vol. 104, no. 8, pp. 1288-1295, 2011.

[17] P. Zhang, Y. Zong, M. Liu, Y. Tai, Y. Cao, and C. Hu, "Prediction outcome in breast cancer patients using test parameters from complete blood count," Molecular and Clinical Oncology, vol. 4, no. 6, pp. 918-924, 2016.

[18] L. Käsmann, L. Bolm, S. E. Schild, S. Janssen, and D. Rades, "Neutrophil-to-Lymphocyte Ratio Predicts Outcome in Limited Disease Small-cell Lung Cancer," Lung, vol. 195, no. 2, pp. 217-224, 2017.

[19] S. L. Highfill, Y. Cui, A. J. Giles et al., "Disruption of CXCR2mediated MDSC tumor trafficking enhances anti-PD1 efficacy," Science Translational Medicine, vol. 6, article 237ra67, no. 237, 2014.

[20] O. Draghiciu, J. Lubbers, H. W. Nijman, and T. Daemen, "Myeloid derived suppressor cells-an overview of combat strategies to increase immunotherapy efficacy," OncoImmunology, vol. 4, no. 1, Article ID 954829, 11 pages, 2015. 


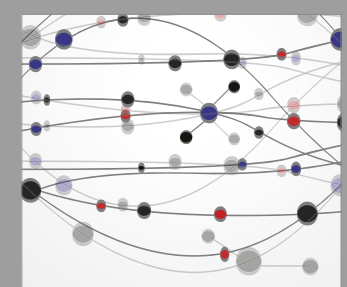

The Scientific World Journal
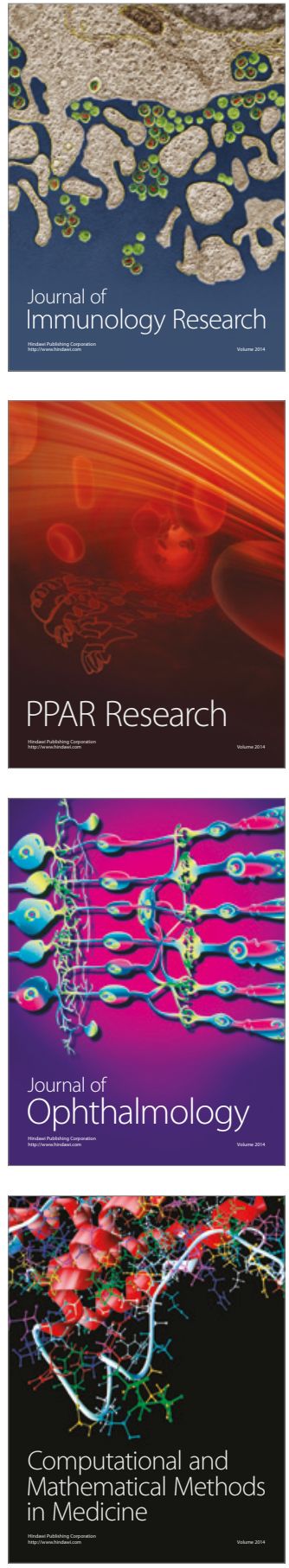

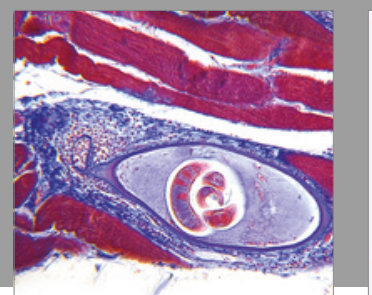

Gastroenterology Research and Practice
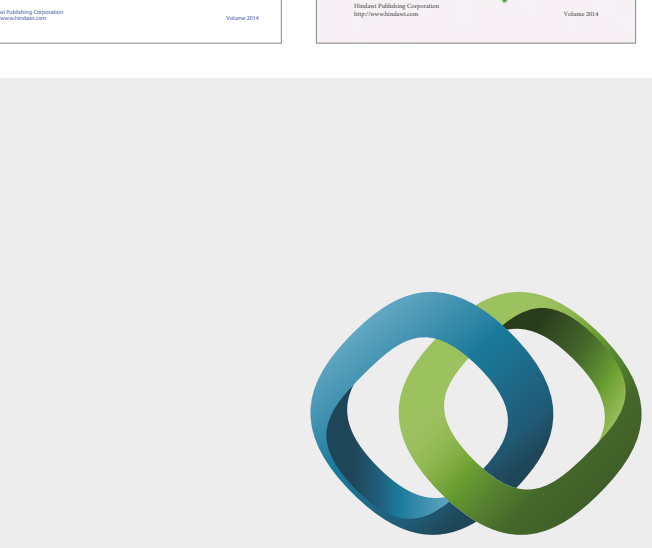

\section{Hindawi}

Submit your manuscripts at

https://www.hindawi.com
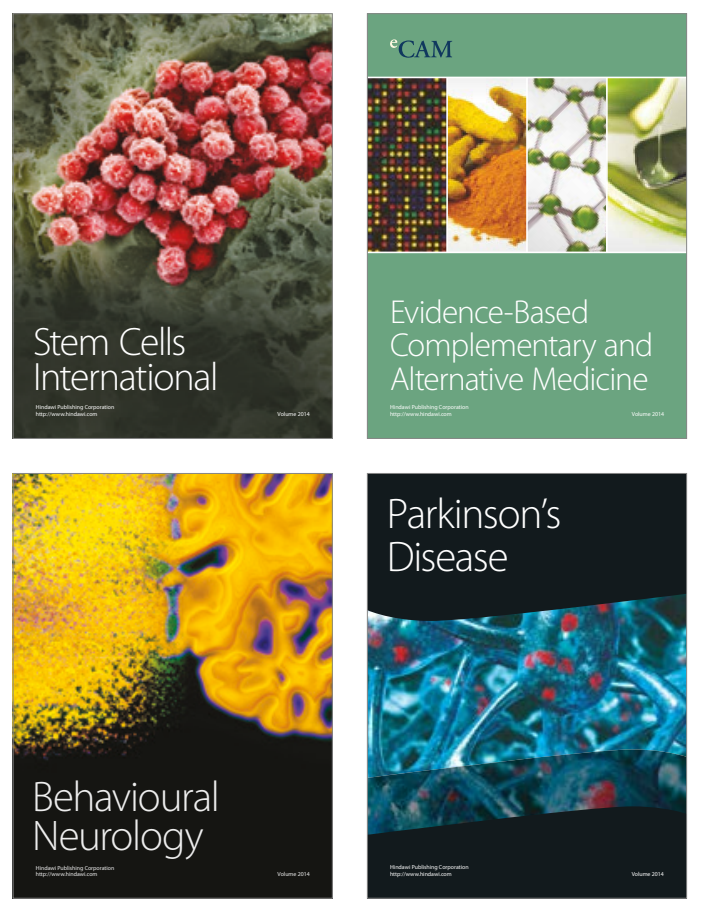
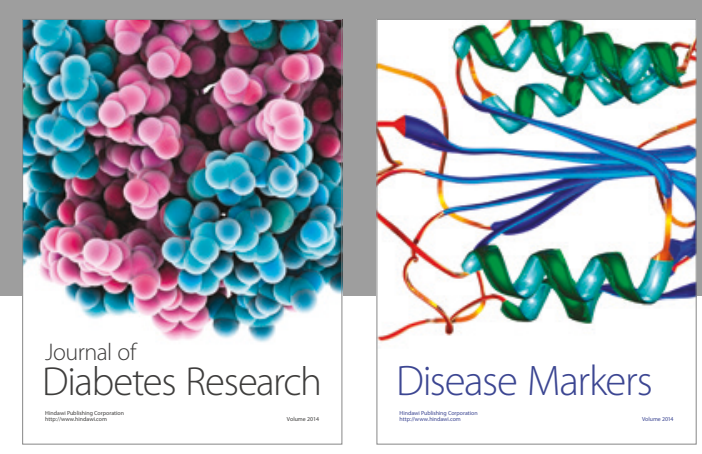

Disease Markers
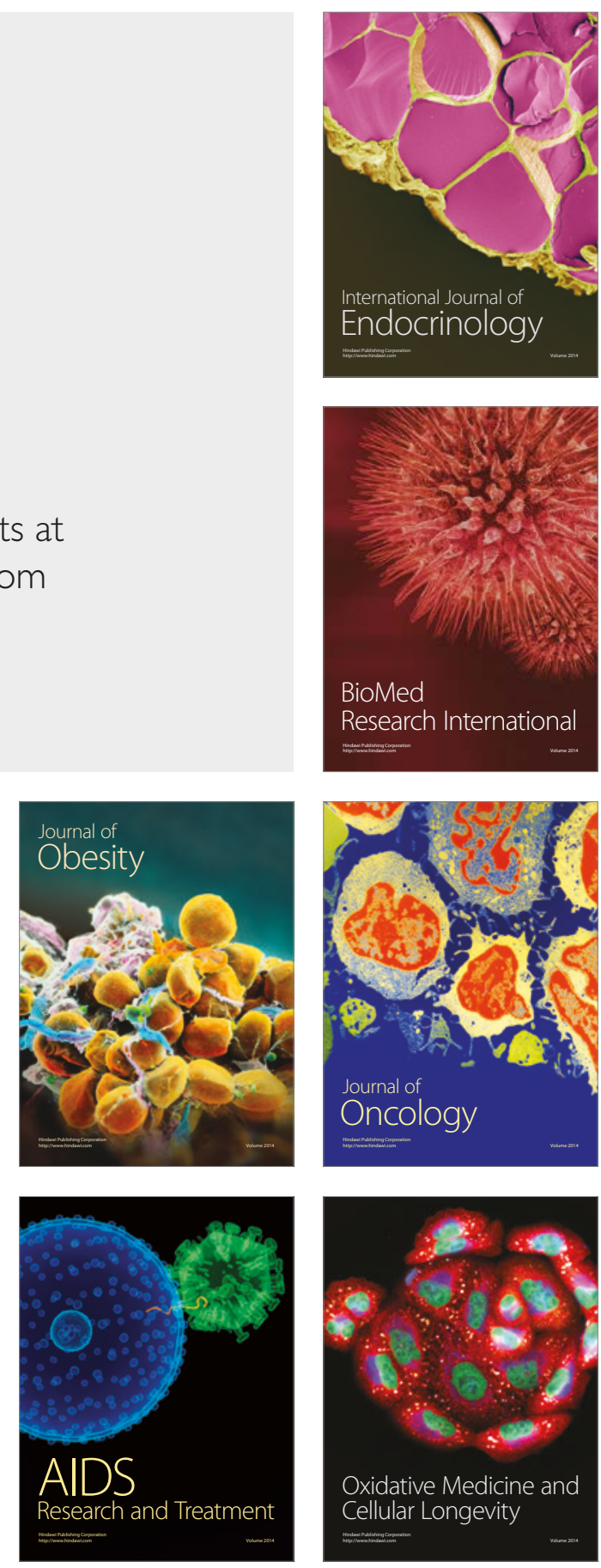